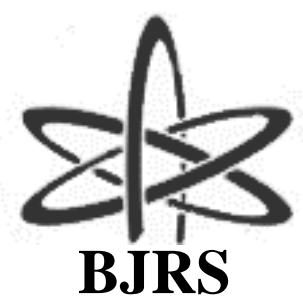

\author{
BRAZILIAN JOURNAL \\ $\mathrm{OF}$ \\ RADIATION SCIENCES \\ 03-01 (2015) 01-07
}

\title{
Avaliação de dose efetiva em um fantoma antropomórfico em situações de emergência radiológica
}

\author{
L.K. Silva ${ }^{\mathrm{a}}$; D.S. Santos ${ }^{\mathrm{a}}$ \\ ${ }^{a}$ Divisão de Dosimetria/Dosimetria Externa, Instituto de Radioproteção e Dosimetria, 22783-127, Rio de Janeiro- \\ RJ, Brasil
}

liviatelecom@hotmail.com

\begin{abstract}
RESUMO
Para o caso de uma estimativa do risco decorrente da exposição de um indivíduo que tenha sido externamente exposto à radiação ionizante, principalmente no que se refere aos efeitos determinísticos e estocásticos à saúde humana, a avaliação de dose efetiva de radiação recebida é uma das informações mais expressivas para essa avaliação. Dessa maneira, devido ao labiríntico sistema utilizado em questão - o corpo humano - essa avaliação deve ser feita utilizando os simuladores físicos, mais conhecidos como fantomas (do inglês, phantoms). A ICRP em sua publicação 110 introduz os fantomas masculino e feminino de Voxel e na publicação 116, apresenta os coeficientes de conversão kerma no ar para dose absorvida em órgãos que se submetam a exposições externas à radiação em um feixe paralelo monoenergético a fótons. Dessa maneira, o presente trabalho pretende realizar uma modelagem da anatomia humana utilizando modelos de Voxels aplicados ao Código Monte Carlo e, através do software Visual Monte Carlo, que simula a irradiação do corpo humano. Fazendo, futuramente, uma avaliação de ambos os resultados com os limites de validade das expressões do TECDOC-1162 da IAEA para o caso de fonte puntiforme.
\end{abstract}

Palavras-chave: Modelos computacionais, Simulação, Dosimetria. 


\section{INTRODUÇÃO}

Na radioproteção quando se deseja fazer uma avaliação da dose em órgãos e para calibração individual utilizam-se, para esses fins, simuladores do corpo humano devido à complexibilidade do sistema. Tais simuladores podem ser: físicos, matemáticos - compostos pela união de sólidos geométricos - ou de voxels.

Neste trabalho, tem-se como objetivo fazer a implementação de um fantoma utilizando o Código de Monte Carlo através do software Visual Monte Carlo para que se possa fazer a avaliação de dose nos indivíduos que foram externamente expostos a qualquer tipo de fonte de radiação ionizante.

\subsection{Os fantomas computacionais}

No fim da década de 60, foram desenvolvidos fantomas mais realistas em paralelo à adoção pela Comissão Internacional de Proteção Radiológica (ICRP) do chamado "Homem Referência” [1]. Em 1969 o comitê Medical Internal Radiation Dose Committee (MIRD) publicou uma descrição de um fantoma composto por elementos geométricos definidos matematicamente para se adequar ao homem referência. Este fantoma ficou conhecido como “fantoma MIRD-5” ou "fantoma de Snyder-Fisher”. Nesta publicação, o fantoma MIRD-5 serviu de base para o uso de métodos de Monte Carlo no cálculo de frações específicas de dose absorvida por órgãos [2].

A ICRP, em sua publicação 110 [3], introduz os fantomas de Voxel, que é uma representação do corpo humano no qual é totalmente descrito através de pequenos elementos de volume chamados voxels, onde estão dispostos em uma matriz tridimensional. Nesta publicação os fantomas representam um adulto padrão masculino e feminino. Já em sua publicação 116 [4], usando tais fantomas de Voxel, apresenta os coeficientes de conversão de kerma no ar para dose absorvida em órgãos para diversos tipos de exposições externas quando o fantoma é exposto a um feixe plano paralelo monoenergético de fótons. Vale ressaltar que é através de uma tomografia computadorizada ou ressonância magnética onde é possível relacionar a cada voxel um tecido do corpo humano. O primeiro fantoma desse tipo a ser utilizado para avaliação de dose efetiva foi desenvolvido por Zubal [5], como na Figura 1. 
Figura 1. Fatia sagital do fantoma de Zubal.

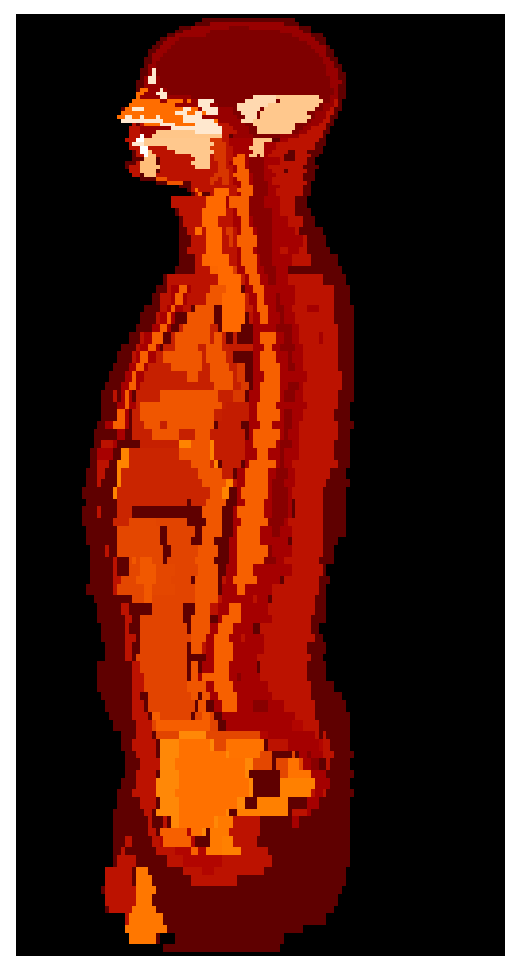

Fonte: Universidade de Washington

\subsection{Visual Monte Carlo}

O Visual Monte Carlo (VMC) foi desenvolvido no Instituto de Radioproteção e Dosimetria, que baseado em fantoma de voxels, simula a irradiação do corpo humano. É usado para estimar a dose efetiva devida à exposição a fontes radioativas [6]. Esta ferramenta de Monte Carlo foi escrita em Visual Basic e permite acompanhar o transporte de fótons emitidos por uma fonte externa puntiforme, nuvem ou solo contaminado. A figura 2 mostra o phantom da versão disponibilizada para download, que permite o cálculo de dose apenas de emissores de fótons. 
Figura 2. Simulação utilizando o software VMC.

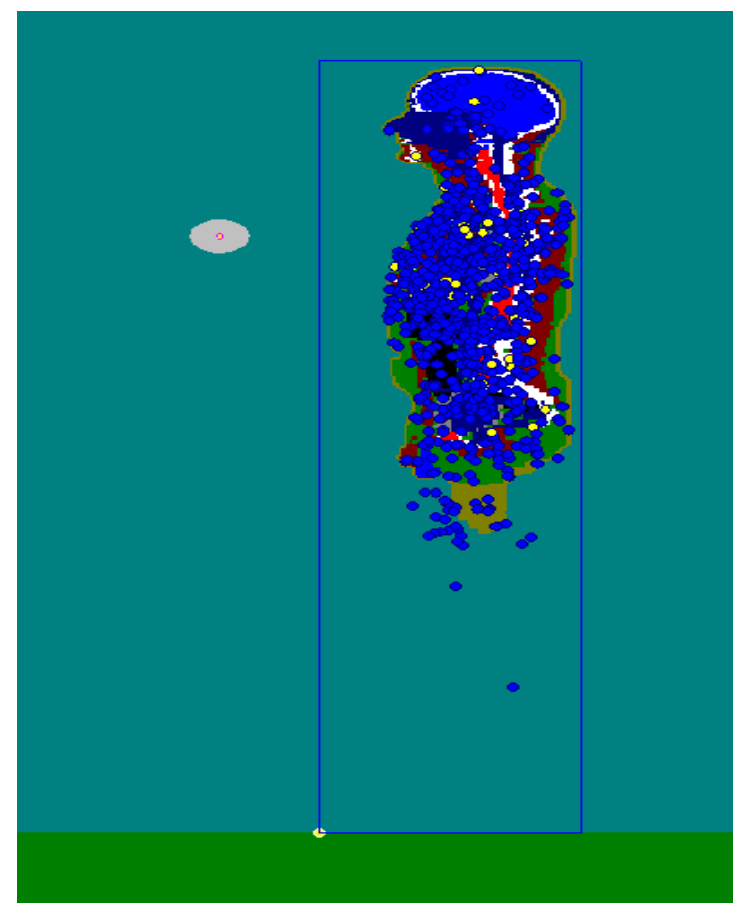

Fonte: Visual Monte Carlo

\subsection{A publicação TECDOC-1162 da IAEA e a expressão para cálculo de dose efetiva}

A Agência Internacional de Energia Atômica (AIEA), em sua publicação TECDOC-1162 [7], apresenta orientações quanto a procedimentos genéricos a serem adotados em situações de emergência radiológica. Como parte destas orientações, recomenda fórmulas para a avaliação de dose efetiva em indivíduos que tenham sido expostos externamente a fontes de radiação em diversas configurações geométricas, para vários radionuclídeos. Essa fórmula, é apresentada abaixo, e relaciona alguns fatores, tais como: atividade da fonte, tempo de exposição distância da fonte e espessura da blindagem, além de um coeficiente que é tabelado e apresentado na própria publicação para vários radionuclídeos.

$$
E_{\text {ext }}=\frac{A \cdot C F_{6} \cdot T_{e} \cdot(0.5)^{\frac{d}{d_{1 / 2}}}}{X^{2}}
$$


Estes coeficientes de conversão são obtidos através do código CONDOS do Laboratório Nacional de Oak Ridge, nos Estados Unidos da América. As expressões para o cálculo de doses efetivas levam em conta a atenuação do campo de radiação por uma possível blindagem da fonte sem, no entanto, considerar efeitos de "build-up" que podem ser significativos. O resultado disto é uma estimativa que em alguns casos é inferior à dose, o que é inadequado para fins de proteção radiológica. Outrossim, não são avaliados os limites de validade destas expressões, que falham quando o indivíduo está muito próximo à fonte de radiação.

\section{MATERIAIS E MÉTODOS}

Em um primeiro momento do trabalho, desenvolveu-se um código de Monte Carlo baseado na ferramenta Geant4 para a avaliação de doses efetivas nos fantomas matemáticos e posteriormente, através da utilização do software VMC, fará a mesma avaliação de dose para os fantomas de voxels, conforme publicado na ICRP 116. Como o caso é de exposição à fótons em uma geometria de fontes puntiformes utiliza-se como fontes radioativas: o ${ }^{137} \mathrm{Cs}$, ${ }^{60} \mathrm{Co}$ e ${ }^{192} \mathrm{Ir}$ para que em seguida possa fazer a validação da simulação com a expressão fornecida no TECDOC1162. Um segundo momento é a avaliação com relação aos efeitos de build up - sempre levando em conta os cortes de produção e acompanhamento de elétrons no Monte Carlo.

\section{RESULTADOS E DISCUSSÕES}

Como visto, a avaliação dos limites de validade do TECDOC-1162 se fará através do posicionamento da fonte puntiforme inicialmente a uma distância grande quando comparada com as dimensões do fantoma. Nesta configuração, espera-se que o resultado do TECDOC-1162 seja muito próximo daquele obtido pelo Monte Carlo, ao aproximar a fonte do fantoma, os dois resultados irão se afastar. A fórmula dada pelo documento é considerada válida enquanto os dois resultados estejam dentro de um limite a ser determinado (resultado do documento sendo da ordem de $20 \%$ ou 30\% diferente do resultado do Monte Carlo). Com relação à avaliação do efeito do build-up, basta envolver a fonte simulada em uma blindagem de materiais diferentes, como por exemplo chumbo, concreto e aço, e avaliar o efeito disto na dose efetiva para as diferentes distâncias da fonte ao fantoma. 


\section{CONCLUSÕES}

Como visto, no desenvolvimento do trabalho espera-se validar a expressão fornecida no TECDOC-1162 para várias distâncias e caso resultado não seja positivo, encontrar a melhor equação para cálculo de dose efetiva para caso emergencial de um acidente radiológico.

\section{AGRADECIMENTO}

Gostaria de agradecer à Comissão Nacional de Energia Nuclear (CNEN) por apoiar-me nesta oportunidade científica. 


\section{REFERÊNCIAS}

1. ICRP - International Commission on Radiation Protection. Report of the Task Group on Reference Man. ICRP Publication 23, Oxford: ICRP, 1975.

2. SNYDER, W.; FORD, M.; WARNER, G.; FISHER, H. Estimates of Absorbed Fractions for Monoenergetic Photon Sources Uniformly Distributed in Various Organs of a Heterogeneous Phantom - MIRD Pamphlet No. 5. J Nucl Med, v. 10, p. 3, 1969.

3. ICRP - International Commission on Radiation Protection. Adult Reference Computational Phantoms. ICRP Publication 110, Oxford: ICRP, p.2, 2009.

4. ICRP - International Commission on Radiation Protection. Conversion Coefficients for Radiological Protection Quantities for External Radiation Exposures. ICRP Publication 116, Oxford: ICRP, P.40, 2010.

5. ZUBAL, I.; HARRELL, C.; SMITH, E.; RATTNER, Z.; GINDI, G; HOFFER, P. Computerized three-dimensional segmented human anatomy. Medical Physics, v. 21, no 2, 1994.

6. HUNT, J. Método de calibração de um sistema de medida in vivo através da simulação matemática da fonte de radiação e do detector. Tese Doutorado. Rio de Janeiro: UERJ, 1998.

7. IAEA - International Atomic Energy Agency. Generic procedures for assessment and response during a radiological emergency. IAEA-TECDOC 1162, Vienna, 2000. 